\title{
Ethnobotanical Survey of Medicinal Plants in kalrayan hills, Eastern Ghats, Tamil Nadu
}

\author{
S. Manikandan*, G. M. Alagu Lakshmanan \\ Department of Botany, Annamalai University, Annamalai Nagar - 608 002, \\ Tamil Nadu, India \\ *E-mail address: gsmani143@gmail.com
}

\begin{abstract}
An ethnomedicinal survey among the kalrayan hills, Salem district, Tamil Nadu during September 2010 to February 2012. A total of 64 species of ethnomedicinal plants belonging to 34 families and 10 species of ethno veterinary plants belonging to 10 families were reported with the help of standard flora among local healers, village head man and elderly tribal persons. The medicinal plants used by village people, tribal and are arranged alphabetically followed by botanical name, family name, local and English name and their therapeutic uses.
\end{abstract}

Keywords: Ethnomedicine; Ethnoveterinary plants; kalrayan hills; Tamil Nadu

\section{INTRODUCTION}

India is a veritable emporium of medicinal and aromatic plants. It has been estimated that out of 15,000 higher plants occurring in India, 9,000 are commonly useful, of which 7,500 are medicinal, 3,900 are edible, 700 are culturally important, 525 are used for fibre, 400 are fodder, 300 for pesticide and insecticide, 300 for gum, resin and dye and 100 for incense and perfume. In terms of the plant materials for traditional medicine, it is estimated that local communities has used over 7,500 plants species. Indian flora has innumerable medicinal plants, which are collected from forest by the tribal villagers. Many of them are being exported to the developed countries. Since ancient time, mankind depended mainly on the plant kingdom to meet its need for medicine, fragrance and flavours. Indian sub-continent is blessed with most varied and diverse soil and climatic conditions, which are suitable for the growth of almost every plant species. Medicinal and aromatic plants and their derivatives to the tune of nearly Rs 200 crores are produced annually in the country. Apart from meeting its domestic requirement, the country exports large quantities of medicinal and aromatic plants and derivatives. There is a considerable scope for India to contribute towards the increasing worldwide demand for medicinal and aromatic plant products. Usage of plants in medicine had been a long practice by man from ancient times. This practice of using plants in medicine is still prevailing not only among the tribals and others living in the rural areas. 


\section{MATERIALS AND METHODS}

\section{1. Study area}

The kalrayan hills is located in the Eastern Ghats range of eastern coast and situated diagonally south east towards Salem in the Villupuram District of Tamil Nadu. The total area is 1000 square kilo metres.

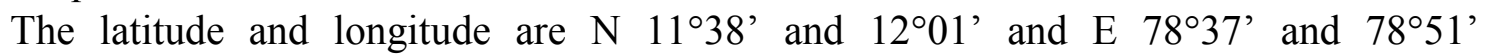
respectively. The hills range from 2000-3000 feet from sea level. This hills present along with Pachamalia, Javadi and Shevaroy hills. The Kalrayan hills divided into two sections such as Northern (Little Kalrayan) and Southern (Big Kalrayan) sections. The Northern Kalrayan average 2100 feet in height while the southern kalrayan average 3000 feet. Some streams are along with hills. They are Manimuktar, Gomuki and Mayura. The most prevalent soil type in this hill ranges from Red loam to black clay is the soil.

The annual temperature in summer seasons (April-June) is $40{ }^{\circ} \mathrm{C}$ and also in winter seasons (November-January) is $11^{\circ} \mathrm{C}$. Total annual rainfall recorded $971 \mathrm{mM}$ at Kallakurichi. According to 2005 census, the population of Kalrayan hills exceeded more than 1,45,000. In which the children population 60,000 .

The Male population 40,000 and the women population 45,000. There are three major types of tribal peoples are living in this area such as Ariya gounder, Kurumba gounder and Jadaya gounder, and who are basically agriculturalists and rearing domestic animals such as cow, goat, sheep, buffalo and pigs. Major livelihoods of tribals are cattle farming, agriculture, Collection of fuel wood and forest resources such as herbal medicines, honey and some edible fruits and tubers from the nearby forests.

\section{2. Methodology}

Field trips ranging from 2 days to a week were made in the study area in every month of the year of study (September 2010 to February 2012) in the Kalrayan hills. The interviews were conducted in the local language in Tamil. Ethnoveterinary information included with the local name of the particular plant, parts utilized, medicinal uses and methods of preparation and administration. The collected ethnoveterinary information was recorded on field note books and plants were identified using the Flora of the Presidency of Madras (J S Gamble 1935), Flora of Tamil Nadu (A. N. Henry and N. C. Nair) and Flora of Tamil Nadu Carnatic (Matthew 1983).

Ethnoveterinary information was gathered from all categories of village people such as the local healers village, head man, elderly persons and the person having a through knowledge of veterinary practices. Some of the commonly occuring veterinary disease were cross checked and conformed with the officials of the local veterinary department. The information gathererd from one place was confirmed by different communities of village people, tribals and ethnic group in different places of investigation.

\section{3. Herborization}

All the species cited as medicinal plants were collected from the field at reproductive stage, with the help of informants in duplicate. A field sheet was recorded with collectors name, vernacular name, local name and ecological parameters. The herbarium samples were dried, processed, identified taxonomically and the names were confirmed with the help of standard flora. 


\section{RESULTS AND DISCUSSION}

The present study revealed that the local people of Kalrayan hills, Salem district, Tamil $\mathrm{Nadu}$, were using 64 species of medicinally important plants belonging to 60 genera and 34 families (Table 1). These medicinally important plants were categorized into five major types. They are herbs (28), shrubs (6), climbers (2) and trees (28). The most medicinally important species were observed in Fabaceae (6), Euphorbiaceae (5) and Rutaceae (4) family. These are commonly occuring medicinally importants plants used to treat 62 types of diseases, and the common diseases like cold, cough, fever, asthma, tuberculosis and as an antidote for poison and in wound healing.

This is the constant with the other general observation which has been reported earlier in relation to medicinal plant studies by the Indian traditional system of medicine like Siddha and Ayurveda (Kirtikar and Basu, 2001; Gogte 2000, Anonymous 1992).

Table 1. Ethnomedicinal plants used by tribals of kalrayan hills in salem district, Tamilnadu.

\begin{tabular}{|c|c|c|c|c|c|}
\hline Plant Name & Family & Local Name & $\begin{array}{c}\text { English } \\
\text { Name }\end{array}$ & Parts Used & Therapeutic uses \\
\hline $\begin{array}{c}\text { Abrus precatorius } \\
\text { L. }\end{array}$ & Fabaceae & Kuntrinmani & Crab's Eye & Root & $\begin{array}{c}\text { Bronchitis, fever, } \\
\text { hepatitis }\end{array}$ \\
\hline $\begin{array}{c}\text { Abutilon indicum } \\
\text { D. }\end{array}$ & Malvaceae & Thuthi & $\begin{array}{l}\text { CountryM } \\
\text { allow }\end{array}$ & Leaves & $\begin{array}{c}\text { Dysentery, } \\
\text { jaundice, piles, } \\
\text { ulcer }\end{array}$ \\
\hline Aegle marmelos L. & Rutaceae & Vilvam & Bael tree & Leaves, Fruit & $\begin{array}{l}\text { Blood sugar } \\
\text { reduction, Skin } \\
\text { boils, Diabetics, } \\
\text { Cold and cough. }\end{array}$ \\
\hline Albizia lebbeck L. & Fabaceae & Vaagai & Siris tree & $\begin{array}{c}\text { Bark, Leaves, } \\
\text { Seed }\end{array}$ & $\begin{array}{l}\text { Toothache, } \\
\text { Antidode, Eye } \\
\text { diseases }\end{array}$ \\
\hline $\begin{array}{c}\text { Aristolochia } \\
\text { bracteolata Lam. }\end{array}$ & Aristolochiaceae & Aduthinnappalai & $\begin{array}{l}\text { Bracteated } \\
\text { Birthwort }\end{array}$ & Root & $\begin{array}{l}\text { Decoction of roots } \\
\text { is used to cure } \\
\text { stomach pain. }\end{array}$ \\
\hline $\begin{array}{c}\text { Artocarphus } \\
\text { heterophyllus L. }\end{array}$ & Moraceae & Pala & Jack fruit & Root, Leaves & $\begin{array}{l}\text { Skin diseases, } \\
\text { Ulcer, Asthma }\end{array}$ \\
\hline $\begin{array}{c}\text { Asparagus } \\
\text { recemosus } \mathrm{W} .\end{array}$ & Liliaceae & $\begin{array}{c}\text { Thannervitan } \\
\text { kizhangu }\end{array}$ & $\begin{array}{c}\text { Indian } \\
\text { asparagus }\end{array}$ & Root & $\begin{array}{c}\text { Diarrhoea, cough, } \\
\text { bronchitis }\end{array}$ \\
\hline $\begin{array}{c}\text { Borassus } \\
\text { flabellifer } \mathrm{L} .\end{array}$ & Arecaceae & Panai & $\begin{array}{l}\text { Palmyra } \\
\text { Palm }\end{array}$ & $\begin{array}{l}\text { Root, young } \\
\text { rachis }\end{array}$ & $\begin{array}{l}\text { Toothache, } \\
\text { toothbrush }\end{array}$ \\
\hline $\begin{array}{c}\text { Borreria } \\
\text { verticillata } \mathrm{L} .\end{array}$ & Rubiaceae & Nathaisoori & $\begin{array}{l}\text { Shaggy } \\
\text { Button } \\
\text { Weed }\end{array}$ & Root & Leucorrhoea \\
\hline $\begin{array}{c}\text { Butea } \\
\text { monosperma L. }\end{array}$ & Fabaceae & Purasu & $\begin{array}{l}\text { Flame of } \\
\text { the Forest }\end{array}$ & Seeds & Ringworm \\
\hline Calamus rotang L. & Arecaceae & Pirambu & Rattan & Tuber & $\begin{array}{c}\text { Cold, Cough and } \\
\text { Fever }\end{array}$ \\
\hline
\end{tabular}




\begin{tabular}{|c|c|c|c|c|c|}
\hline $\begin{array}{c}\text { Calotropis procera } \\
\text { A. }\end{array}$ & Earukku & Velleruku & Milk Weed & Bark, Flowers & $\begin{array}{c}\text { Dysentry, Cold, } \\
\text { Cough and Asthma }\end{array}$ \\
\hline $\begin{array}{l}\text { Calophyllum } \\
\text { inophyllum } \mathrm{L} .\end{array}$ & Guttiferae & Punnai & $\begin{array}{l}\text { Indian } \\
\text { Laurel }\end{array}$ & Seeds & Scabies \\
\hline $\begin{array}{c}\text { Capparis } \\
\text { divaricata } \mathrm{L} .\end{array}$ & Capparaceae & Thoratti & $\begin{array}{l}\text { Indian } \\
\text { Caper }\end{array}$ & Bark, Leaves & $\begin{array}{c}\text { Dysentery, } \\
\text { Stomach Problems }\end{array}$ \\
\hline $\begin{array}{l}\text { Cardiospermum } \\
\text { halicacabum L. }\end{array}$ & Sapindaceae & Mudakkaruthaan & $\begin{array}{l}\text { Ballon } \\
\text { Vine }\end{array}$ & Leaves & $\begin{array}{l}\text { Plant leaf extract } \\
\text { reduces body pain } \\
\text { and decoction of } \\
\text { whole plant is used } \\
\text { for curing } \\
\text { rheumatism. }\end{array}$ \\
\hline $\begin{array}{c}\text { Cassia auriculata } \\
\text { L. }\end{array}$ & Caesalpiniaceae & Aavarai & $\begin{array}{l}\text { Tanner's } \\
\text { Cassia }\end{array}$ & $\begin{array}{l}\text { Young stem, } \\
\text { Leaves }\end{array}$ & $\begin{array}{l}\text { Toothbrush, } \\
\text { Stomach ulcer. }\end{array}$ \\
\hline Cassia fistula L. & Caesalpiniaceae & Konrai & $\begin{array}{l}\text { Golden } \\
\text { Shower }\end{array}$ & Young leaves & $\begin{array}{c}\text { Foetid smell of } \\
\text { mouth. }\end{array}$ \\
\hline $\begin{array}{l}\text { Cissampelos } \\
\text { pareira } \mathrm{L} .\end{array}$ & Menispermaceae & Ponmusutai & $\begin{array}{l}\text { Velvet- } \\
\text { Leaf } \\
\text { Pareira }\end{array}$ & Root, leaves & $\begin{array}{l}\text { Fistula, Antidode, } \\
\text { Blood purification }\end{array}$ \\
\hline $\begin{array}{c}\text { Cissus } \\
\text { quadrangularis L. }\end{array}$ & Vitaceae & Pirantai & $\begin{array}{l}\text { Adamant } \\
\text { Creeper }\end{array}$ & Whole plant & $\begin{array}{l}\text { Stem and leaf paste } \\
\text { used to cure bone } \\
\text { fracture and root } \\
\text { paste used for gas } \\
\text { trouble. }\end{array}$ \\
\hline Clausena dentate & Rutaceaec & Aana & --- & Fruit & Rarely edible \\
\hline $\begin{array}{l}\text { Coccinia grandis } \\
\text { (L.) Voigt }\end{array}$ & Cucurbitaceae & Kovai & Ivy-Gourd & Whole plant & $\begin{array}{c}\text { Leaves juice taken } \\
\text { for internally for } \\
\text { ulcer. }\end{array}$ \\
\hline $\begin{array}{c}\text { Dodonaea viscosa } \\
\mathrm{J} .\end{array}$ & Sapindaceae & Virali & $\begin{array}{c}\text { Jamacia } \\
\text { Switch } \\
\text { Sorrel }\end{array}$ & Leaves & Wounds, Swelling \\
\hline Euphorbia hirta L. & Euphorbiaceae & Amman patcharisi & $\begin{array}{l}\text { Pill- } \\
\text { bearing } \\
\text { Spurge }\end{array}$ & $\begin{array}{l}\text { Leaves, } \\
\text { flower, fruits }\end{array}$ & $\begin{array}{c}\text { Asthma, respiratory } \\
\text { infections }\end{array}$ \\
\hline $\begin{array}{c}\text { Evolvulus } \\
\text { alsinoides L. }\end{array}$ & Convolvulaceae & Vishnukaranthai & --- & Whole plant & $\begin{array}{l}\text { Brain disorders, } \\
\text { epilepsy, nervous } \\
\text { problems }\end{array}$ \\
\hline Ficus mollis $\mathrm{V}$. & Moraceae & Kal-Athi & --- & Bark & Urinary infections \\
\hline Ficus religiosa L. & Moraceae & Arasu & Bot-tree & Leaves & $\begin{array}{l}\text { Latex is given to } \\
\text { children in fever } \\
\text { and dullness. }\end{array}$ \\
\hline Garcinia indica $\mathrm{C}$. & Guttiferae & Pazhampuli & $\begin{array}{c}\text { Kokam } \\
\text { Butter tree }\end{array}$ & Leaves & $\begin{array}{l}\text { Cosmetic } \\
\text { ingredients }\end{array}$ \\
\hline Gmilina arborea & Verbinaceae & Kumala & $\begin{array}{c}\text { Candahar } \\
\text { tree }\end{array}$ & Root Bark & $\begin{array}{l}\text { Impotency, Prolong } \\
\text { coitus time. }\end{array}$ \\
\hline $\begin{array}{l}\text { Indigofera } \\
\text { tinctoria } \mathrm{L} .\end{array}$ & Fabaceae & Avuri, Neeli & Indigo & Leaves & $\begin{array}{l}\text { Used in bronchitis, } \\
\text { dry cough, } \\
\text { respiratory } \\
\text { infections, } \\
\text { tuberculosis. }\end{array}$ \\
\hline
\end{tabular}




\begin{tabular}{|c|c|c|c|c|c|}
\hline Ixora bractiata L. & Rubiaceae & Thetti & --- & Root Bark & $\begin{array}{l}\text { Root bark paste } \\
\text { with coconut pulp } \\
\text { applied for } \\
\text { inflammation. }\end{array}$ \\
\hline Jatropha curcas L. & Euphorbiaceae & Kaatu-amanakku & Physic Nut & Whole plant & $\begin{array}{l}\text { Roots are poultice } \\
\text { for fractures } \\
\text { Seeds are purgative } \\
\text { treats diarrhoea. }\end{array}$ \\
\hline $\begin{array}{c}\text { Limonia } \\
\text { acidissima L. }\end{array}$ & Rutaceae & Vila & --- & Bark & $\begin{array}{c}\text { Insect bites, } \\
\text { diarrhoea, } \\
\text { dysentery, snake } \\
\text { bite. }\end{array}$ \\
\hline Macranga peltata & Euphorbiaceae & Vaadha neeki & --- & Wood & As cheap timber. \\
\hline $\begin{array}{c}\text { Madhuca } \\
\text { longifolia } \mathrm{J} .\end{array}$ & Sapotaceae & Iluppai & $\begin{array}{l}\text { South } \\
\text { Indian } \\
\text { Mahua }\end{array}$ & Bark & Skin diseases \\
\hline $\begin{array}{c}\text { Mangifera indica } \\
\text { L. }\end{array}$ & Anacardiaceae & Maa & Mango & Bark & $\begin{array}{l}\text { Gargle for mouth } \\
\text { ulcer, foetid smell. }\end{array}$ \\
\hline $\begin{array}{c}\text { Melia azedarach } \\
\text { L. }\end{array}$ & Meliaceae & Malai vembu & $\begin{array}{l}\text { Persian } \\
\text { Lilac }\end{array}$ & $\begin{array}{l}\text { Leaves, Seed } \\
\text { oil, bark }\end{array}$ & $\begin{array}{l}\text { Small box, viral } \\
\text { fever, skin } \\
\text { infections, bark } \\
\text { extracts to control } \\
\text { women hormone } \\
\text { problems, } \\
\text { antiseptic. }\end{array}$ \\
\hline $\begin{array}{c}\text { Melothria } \\
\text { maderaspatana L. }\end{array}$ & Cucurbitaceae & Musumusukai & --- & Leaves & Allergic, Asthma \\
\hline $\begin{array}{c}\text { Michelia } \\
\text { champaca } \mathrm{L} \text {. }\end{array}$ & Magnoliaceae & Senpagam & $\begin{array}{l}\text { Golden } \\
\text { Champa }\end{array}$ & $\begin{array}{l}\text { Bark, Leaves, } \\
\text { Flowers }\end{array}$ & $\begin{array}{l}\text { Cold, Fever, } \\
\text { Stomach ache, } \\
\text { Urinary problems }\end{array}$ \\
\hline Mimosa pudica L. & Mimosaceae & Thottasuringi & $\begin{array}{l}\text { Sensitive- } \\
\text { plant }\end{array}$ & Leaves & $\begin{array}{c}\text { Used to Blood } \\
\text { purifier, diarrhoea, } \\
\text { dysentery. }\end{array}$ \\
\hline $\begin{array}{c}\text { Mimusops elengi } \\
\text { L. }\end{array}$ & Sapotaceae & Mahizham & $\begin{array}{l}\text { Spanish- } \\
\text { Cherry }\end{array}$ & $\begin{array}{c}\text { Bark, Leaves, } \\
\text { Flowers }\end{array}$ & $\begin{array}{c}\text { Toothache, Uterus } \\
\text { problems }\end{array}$ \\
\hline Mucuna puriens L. & Fabaceae & Poonaicali & $\begin{array}{c}\text { Horse-eye } \\
\text { Bean. }\end{array}$ & Seed & $\begin{array}{l}\text { Male sterility, } \\
\text { Nervous diseases }\end{array}$ \\
\hline $\begin{array}{c}\text { Murraya } \\
\text { paniculata }\end{array}$ & Rutaceae & $\begin{array}{c}\text { Kattu } \\
\text { karuveppilai }\end{array}$ & --- & Root, Leaves & $\begin{array}{c}\text { Dropsy, body ache, } \\
\text { fever, } \\
\text { inflammation. }\end{array}$ \\
\hline $\begin{array}{c}\text { Ocimum sanctum } \\
\text { L. }\end{array}$ & Lamiaceae & Thulasi & $\begin{array}{c}\text { Sacred } \\
\text { Basil }\end{array}$ & Leaves & $\begin{array}{l}\text { Common cold, } \\
\text { weakness, stress, to } \\
\text { treat coughs in } \\
\text { children. }\end{array}$ \\
\hline $\begin{array}{l}\text { Pergularia daemia } \\
\text { (Forrsk.) Chiov. }\end{array}$ & Asclepiadaceae & Uthamani & $\begin{array}{l}\text { Hairknot } \\
\text { Plant }\end{array}$ & Leaves & $\begin{array}{l}\text { Leaves juice used } \\
\text { for urinary } \\
\text { problems, fever, } \\
\text { asthma and gas } \\
\text { trouble. }\end{array}$ \\
\hline $\begin{array}{l}\text { Phoenix sylvestris } \\
\text { L. }\end{array}$ & Arecaceae & Echam & $\begin{array}{l}\text { Wild Date } \\
\text { Palm }\end{array}$ & Root & Toothache \\
\hline
\end{tabular}




\begin{tabular}{|c|c|c|c|c|c|}
\hline $\begin{array}{c}\text { Phyllanthus } \\
\text { amarus Schum. \& } \\
\text { Thonn. } \\
\end{array}$ & Euphorbiaceae & Kizha-Nelli & --- & Whole Plant & $\begin{array}{c}\text { Remedy against } \\
\text { scabies, viral } \\
\text { hepatitis, Jaundice. }\end{array}$ \\
\hline $\begin{array}{l}\text { Phyllanthus } \\
\text { emblica L. }\end{array}$ & Euphorbiaceae & Nelli & --- & $\begin{array}{c}\text { Root bark, } \\
\text { Leaves, Fruits }\end{array}$ & $\begin{array}{l}\text { Fever, Mouth ulcer, } \\
\text { Blood pressure }\end{array}$ \\
\hline Piper betle L. & Piperaceae & Vetrilai & $\begin{array}{c}\text { Betel } \\
\text { pepper }\end{array}$ & Leaves & Digestive \\
\hline $\begin{array}{c}\text { Portulaca } \\
\text { quadrifida } \mathrm{L} .\end{array}$ & Portulacaceae & Siru pasalai keerai & & Leaves & $\begin{array}{l}\text { Plant decoction is } \\
\text { good for cough. }\end{array}$ \\
\hline $\begin{array}{l}\text { Pterocarpus } \\
\text { marsupium L. }\end{array}$ & Fabaceae & Vengai & $\begin{array}{l}\text { Indian } \\
\text { Kino tree }\end{array}$ & $\begin{array}{l}\text { Bark, Flowers, } \\
\text { Gum }\end{array}$ & Fever, Toothache \\
\hline $\begin{array}{c}\text { Punica granatum } \\
\text { L. }\end{array}$ & Lythraceae & Madhulai & $\begin{array}{l}\text { Pomegra- } \\
\text { nate }\end{array}$ & Fruit & $\begin{array}{l}\text { Dysentery, } \\
\text { Diarrhoea }\end{array}$ \\
\hline Santalum album L. & Santalaceae & Santhanam & Santal & Wood & $\begin{array}{l}\text { Pimples, Urinary } \\
\text { infections }\end{array}$ \\
\hline Saraca asoca D. & Fabaceae & Asogu & $\begin{array}{c}\text { Fake } \\
\text { Asoka tree }\end{array}$ & Bark, Flowers & $\begin{array}{c}\text { Skin disease, } \\
\text { Dysentery }\end{array}$ \\
\hline $\begin{array}{l}\text { Senna sophera (L.) } \\
\text { Roxb. }\end{array}$ & Caesalpiniaceae & Ponnavaarai & $\begin{array}{l}\text { Tinnivelly } \\
\text { senna }\end{array}$ & Leaves & $\begin{array}{l}\text { Used against } \\
\text { ringworm and } \\
\text { scabies. }\end{array}$ \\
\hline Sida cardifolia L. & Malvaceae & Sitramuti & $\begin{array}{l}\text { Country } \\
\text { Mallow }\end{array}$ & Leaves & $\begin{array}{c}\text { Stomatitis, } \\
\text { Asthmatic } \\
\text { bronchitis, Nervous } \\
\text { disorders }\end{array}$ \\
\hline $\begin{array}{c}\text { Solanum } \\
\text { virgianum L. }\end{array}$ & Solanaceae & Kanndankathari & --- & Fruit & $\begin{array}{c}\text { Unripe fruits are } \\
\text { eaten to cure } \\
\text { cough. }\end{array}$ \\
\hline $\begin{array}{l}\text { Strychnos nux- } \\
\text { vomica } \mathrm{L} .\end{array}$ & Loganiaceae & Eatti & $\begin{array}{c}\text { Nux } \\
\text { vomica }\end{array}$ & Leaves & Body boils \\
\hline Syzygium cuminii & Myrtaceae & Naval & $\begin{array}{l}\text { Black } \\
\text { Plum } \\
\end{array}$ & $\begin{array}{c}\text { Bark, Seed, } \\
\text { Leaves } \\
\end{array}$ & $\begin{array}{l}\text { Dysentry, diabetics, } \\
\text { antihelmintic fever. }\end{array}$ \\
\hline $\begin{array}{c}\text { Tephrosia } \\
\text { purpurea L. }\end{array}$ & Fabaceae & Kolinchi & $\begin{array}{l}\text { Wild } \\
\text { Indigo }\end{array}$ & Whole Plant & $\begin{array}{l}\text { Kidney, liver } \\
\text { diseases }\end{array}$ \\
\hline $\begin{array}{c}\text { Terminalia } \\
\text { bellirica }\end{array}$ & Combretaceae & Thandri & $\begin{array}{c}\text { Belleric } \\
\text { Myrobalan }\end{array}$ & Leaves, Fruit & $\begin{array}{l}\text { Infertility, } \\
\text { Diabetics }\end{array}$ \\
\hline $\begin{array}{l}\text { Terminalia } \\
\text { catappa } \mathrm{L} .\end{array}$ & Combretaceae & Padam & $\begin{array}{l}\text { Indian } \\
\text { Almond }\end{array}$ & Gum & Cough, Dysentry \\
\hline $\begin{array}{c}\text { Tribulus terrestris } \\
\text { L. }\end{array}$ & Zygophyllaceae & Nerunjil & $\begin{array}{c}\text { Puncture } \\
\text { Vine }\end{array}$ & Root & $\begin{array}{c}\text { Urinary stones, } \\
\text { infections. }\end{array}$ \\
\hline Tylophora indica & Asclepiadaceae & Nancharuppan & $\begin{array}{c}\text { Emetic } \\
\text { Swallow } \\
\text { Wort } \\
\end{array}$ & Root, Leaves & Asthma, Antidode \\
\hline $\begin{array}{c}\text { Withania } \\
\text { somnifera }(\mathrm{L} .) \\
\text { Dunal }\end{array}$ & Solanaceae & Ashwakantha & $\begin{array}{l}\text { Winter } \\
\text { Cherry }\end{array}$ & Root & $\begin{array}{l}\text { Root paste is } \\
\text { applied externally } \\
\text { for ulcers, } \\
\text { inflammatory } \\
\text { conditions and } \\
\text { scabies. }\end{array}$ \\
\hline
\end{tabular}

In other side most of the local healers and peoples are using the ethnoveterinary medicinal species (Table 2) such as Cassia tora L. (Ceasalpinaceae), Cissus quadrangularis 
L. (Vitaceae), Citrullus colosynthis L. (Cucurbitaceae), Dalbergia latifolia Roxb. (Fabaceae), Dendrocalamus strictus Nees. (Poaceae), Lannea coramandalica Merr. (Anacardiaceae), Leucas aspera L. (Lamiaceae), Pedalium murex L. (Pedaliaceae), Taddalia asiatica Lam. (Rutaceae) and Wattakaka volubilis L. (Asclepiadaceae). These plants were used for the treatement of skin diseases, fever, cough, worms and swellings in cow, goat and pigs.

Table 2. Ethnoveterinary plants and their therapeutic uses.

\begin{tabular}{|c|c|c|c|c|c|}
\hline Botanical Name & Family & Local Name & $\begin{array}{l}\text { English } \\
\text { Name }\end{array}$ & $\begin{array}{c}\text { Animals } \\
\text { treated }\end{array}$ & Therapeutic Uses \\
\hline Cassia tora $\mathrm{L}$. & Ceasalpinaceae & Usithagarai & $\begin{array}{l}\text { Sickle } \\
\text { Senna }\end{array}$ & Cow, goat & $\begin{array}{l}\text { Seed is mixed with } \\
\text { water and ground into } \\
\text { paste and applied } \\
\text { topically to cure skin } \\
\text { diseases. }\end{array}$ \\
\hline $\begin{array}{c}\text { Cissus } \\
\text { quadrangularis L. }\end{array}$ & Vitaceae & Pirantai & $\begin{array}{l}\text { Adamant } \\
\text { Creeper }\end{array}$ & Goat & $\begin{array}{l}\text { Leaves are ground } \\
\text { with pepper and } \\
\text { garlic and made into } \\
\text { a decoction. The } \\
\text { decoction is given } \\
\text { to cure fever. }\end{array}$ \\
\hline $\begin{array}{c}\text { Citrullus colosynthis } \\
\text { L. }\end{array}$ & Cucurbitaceae & Varikurumathai & $\begin{array}{c}\text { Colocynth } \\
\text { Bitter } \\
\text { Apple }\end{array}$ & Cow, goat & $\begin{array}{l}\text { Root is ground with } \\
\text { water and the } \\
\text { decoction obtained is } \\
\text { given to cure cough. }\end{array}$ \\
\hline $\begin{array}{l}\text { Dalbergia latifolia } \\
\text { Roxb. }\end{array}$ & Fabaceae & Eettimaram & $\begin{array}{l}\text { East Indian } \\
\text { Rosewood }\end{array}$ & Cow, goat & $\begin{array}{l}\text { Stem bark is ground } \\
\text { with garlic and } \\
\text { pepper and the } \\
\text { mixture is given for } \\
\text { the animals which are } \\
\text { lazy in grazing. }\end{array}$ \\
\hline $\begin{array}{l}\text { Dendrocalamus } \\
\text { strictus Nees. }\end{array}$ & Poaceae & Kalmungil & --- & Cow & $\begin{array}{l}\text { Roasted fruits are } \\
\text { given once a day to } \\
\text { treat dysentery and } \\
\text { cough until cure. }\end{array}$ \\
\hline $\begin{array}{c}\text { Lannea } \\
\text { coramandalica Merr. }\end{array}$ & Anacardiaceae & Uthiyamaram & --- & Pigs & $\begin{array}{l}\text { Stem bark is ground } \\
\text { with ginger and garlic } \\
\text { and the paste is given } \\
\text { to cure fever }\end{array}$ \\
\hline Leucas aspera L. & Lamiaceae & Thumbai & $\begin{array}{l}\text { White } \\
\text { Dead } \\
\text { Nettle }\end{array}$ & Cow & $\begin{array}{l}\text { The leaf juice used to } \\
\text { cure cut wounds and } \\
\text { worms }\end{array}$ \\
\hline Pedalium murex $\mathrm{L}$. & Pedaliaceae & Perunerunjil & Caltrops & Cow & $\begin{array}{c}\text { Leaf is ground with } \\
\text { ginger and common } \\
\text { salt and given to cure } \\
\text { fever }\end{array}$ \\
\hline $\begin{array}{l}\text { Toddalia asiatica } \\
\text { Lam. }\end{array}$ & Rutaceae & Milagaranai & $\begin{array}{l}\text { Forest } \\
\text { Pepper }\end{array}$ & Cow, goat & $\begin{array}{l}\text { Leaf is mixed with } \\
\text { onion, pepper and } \\
\text { garlic, ground into a } \\
\text { paste and given to } \\
\text { cure swellings. }\end{array}$ \\
\hline $\begin{array}{l}\text { Wattakaka volubilis } \\
\text { (L. f.) Stapf. }\end{array}$ & Asclepiadaceae & Perunkurinjan & --- & Cow, goat & $\begin{array}{l}\text { Leaf paste is mixed } \\
\text { with common salt } \\
\text { and applied on } \\
\text { affected places to } \\
\text { treat all types of } \\
\text { swellings. }\end{array}$ \\
\hline
\end{tabular}


Different types of preparations were made from medicinally important plants include decoction, juice, powder, oil, paste and whole plant extract. Some plants were even used in more than one form of preparations. Majority of the plants preparation were in the form of decoction obtained from the roots, seeds, stems, leaves and flowers. In this, the leaves are the predominant part utilized in ethnomedicinal treatements.

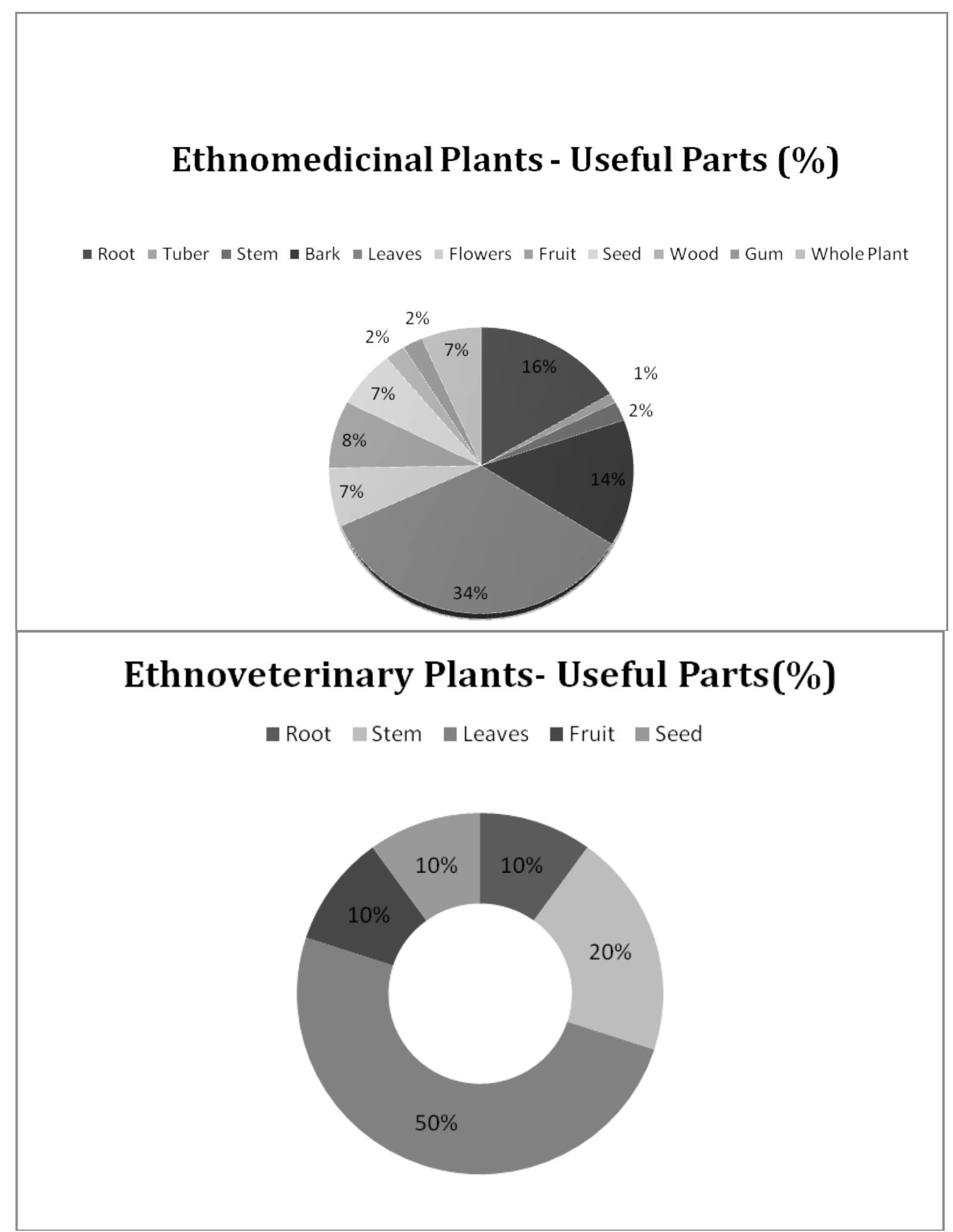




\section{Types of Preparations (\%)}

Decoction Juice Paste Infusion Food supplement
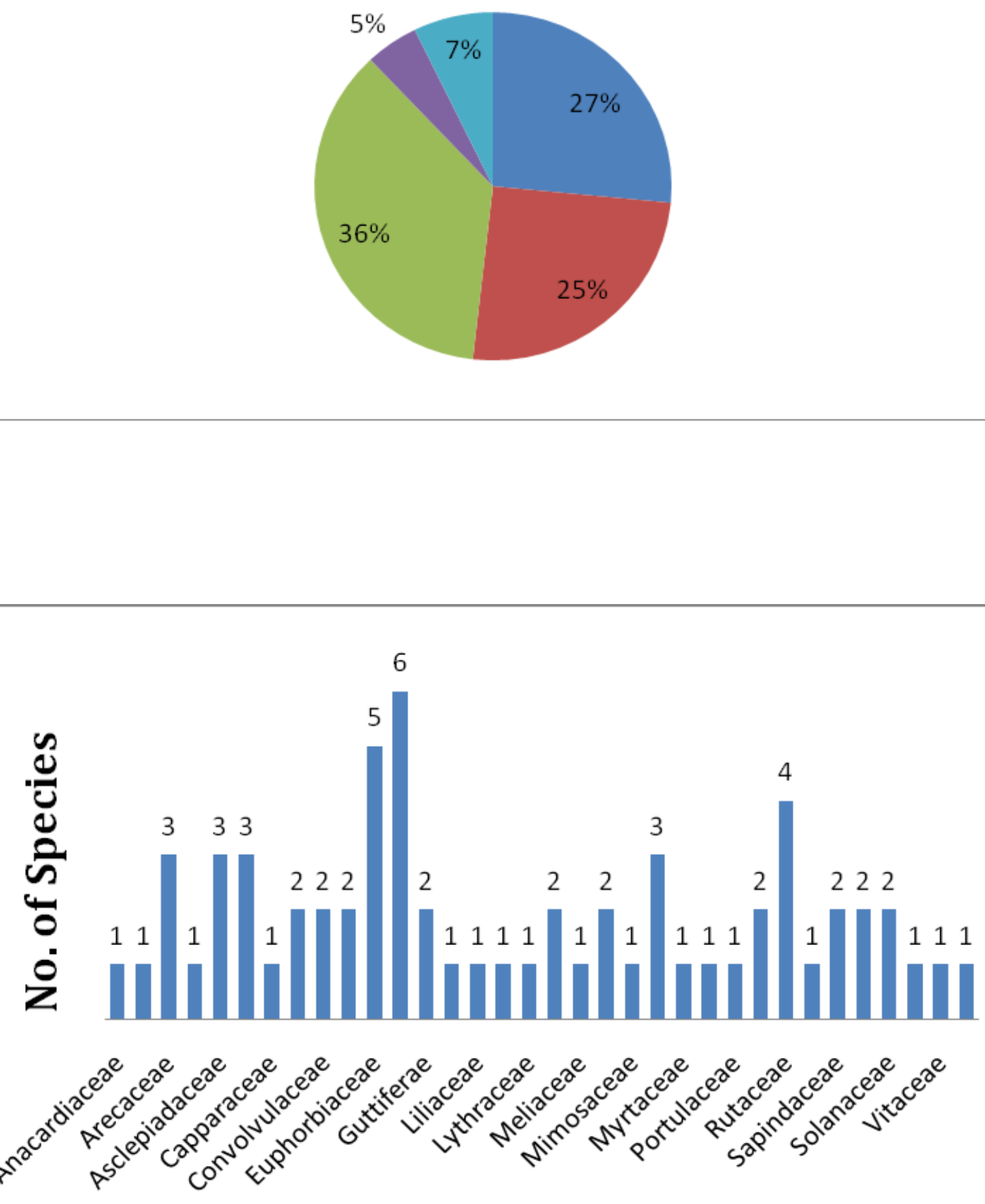

Name of the families 


\section{CONCLUSIONS}

Tribal knowledge of plants in many tribal communities is changing because of rapid socioeconomic and cultural changes. Documentation of this knowledge is valuable for the communities and their future generations and for scientific consideration of wider uses of traditional knowledge in treating domestic animals. The low cost and almost no side effects of these traditional preparations with medicinal plants make them adaptable by the local community. The wealth of this tribal knowledge of medicinal plants points to a great potential for research and the discovery of new drugs to cure the diseases of animals. So, further scientific assesment of these medicines for phytochemical, biological and pre-clinical and clinical studies is, however, greatly needed.

Basically, medicinal plants play an important role in providing knowledge to the researches in the field of ethnobotany and ethnopharmacology. The observations of present study showed that traditional medicine plays a significant role among the local healers and people of kalrayan hills.

\section{References}

[1] Anonymous, Ethnobotany in India, A Status report, All India coordinated research project in ethnobotany, Ministry of Environment Forests, Government of India, New Delhi, 1994.

[2] Anonymous, Annual Report, Indian Council of Forestry Research and Education, Dehradun, 1992-1993, 193-207.

[3] Anonymous, Envis Newslett 1(2) (2002) 5.

[4] Anonymous, The Useful Plants of India, Publications and Information Directorate, New Delhi, 1992.

[5] Anonymous, Ethnobotany in India, A Status report, All India coordinated research project in ethnobotany, Ministry of Environment Forests, Government of India, New Delhi, 1994.

[6] Gamble J. S., Fischer C. E. C., The flora of the Presidency of Madras, Reprinted Edition, Vol. I-III, (Botanical Survey of India, Calcutta), 1959.

[7] Henry A. N., Kumari G. R., Chitra V., Flora of Tamil Nadu, India, Series I, Vol II \& III, Botanical Survey of India, Southern Circle, Coimbatore, 1987.

[8] Nair N. C., Henry A. N., Flora of Tamil Nadu, India, Series I, Vol I, Botanical Survey of India, Southern Circle, Coimbatore, 1983.

[9] Mathew K. M., An excursion flora of Tamil Nadu, India, Oxford and IBH Publishing Co. Ltd., New Delhi, 1991.

[10] Jain S. K., Goel A. K., A Manual of Ethnobotany, (ed.) S K Jain, (Scientific Publishers, Jodhpur), 1995; 142.

[11] Binu Thomas A., et al, Int. Journal of Biological Tech. 2(2) (2011) 72-75.

[12] Ganesan S., et al, Indian Journal of Traditional Knowledge 7(2) (2008) 347-354.

[13] Selvaraju A et al, Medicinal Plants 3(3) (2011). 
[14] Sankaranarayan S., et al, J. Med. Plants Research 4(12) (2010) 1089-1101.

[15] Balakrishnan V., et al, Global Journal of Pharmacology 3(1) (2009) 15-23.

[16] Mutheeswaran S., et al, Journal of Ethnopharmacology 137 (2011) 523-533.

[17] S. Dhanam, International Letters of Natural Sciences 11(2) (2014) 197-208. 